\title{
Visualization of Gut Microbiota-host Interactions via Fluorescence In Situ Hybridization, Lectin Staining, and Imaging
}

\author{
Katharine M. $\mathbf{N g}^{1,2}$, Carolina Tropini ${ }^{1,2,3}$ \\ ${ }^{1}$ School of Biomedical Engineering, University of British Columbia ${ }^{2}$ Department of Microbiology and Immunology, University of British Columbia ${ }^{3}$ Humans \\ and the Microbiome Program, Canadian Institute for Advanced Research (CIFAR)
}

\section{Corresponding Author}

Carolina Tropini

carolina.tropini@ubc.ca

\section{Citation}

Ng, K.M., Tropini, C. Visualization of Gut Microbiota-host Interactions via Fluorescence In Situ Hybridization, Lectin Staining, and Imaging. J. Vis. Exp. (173), e62646, doi:10.3791/62646 (2021).

\section{Date Published}

July 9,2021

DOI

$10.3791 / 62646$

URL

jove.com/video/62646

\section{Abstract}

Measuring the localization of microbes within their in vivo context is an essential step in revealing the functional relationships between the microbiota and the vertebrate gut. The spatial landscape of the gut microbiota is tightly controlled by physical features - intestinal mucus, crypts, and folds - and is affected by host-controlled properties such as $\mathrm{pH}$, oxygen availability, and immune factors. These properties limit the ability of commensal microbes and pathogens alike to colonize the gut stably. At the micron-scale, microbial organization determines the close-range interactions between different microbes as well as the interactions between microbes and their host. These interactions then affect large-scale organ function and host health.

This protocol enables the visualization of the gut microbiota spatial organization from distances between cells to organ-wide scales. The method is based on fixing gut tissues while preserving intestinal structure and mucus properties. The fixed samples are then embedded, sectioned, and stained to highlight specific bacterial species through fluorescence in situ hybridization (FISH). Host features, such as mucus and host cell components, are labeled with fluorescently labeled lectins. Finally, the stained sections are imaged using a confocal microscope utilizing tile-scan imaging at high magnification to bridge the micron to centimeter length scales. This type of imaging can be applied to intestinal sections from animal models and biopsies from human tissues to determine the biogeography of the microbiota in the gut in health and disease.

\section{Introduction}

Microbial visualization techniques have origins that are as old as microbiology itself, when Antonie Van Leeuwenhoek used his microscope to observe bacteria (which he called "animalcules") from tooth plaque and stool in the $17^{\text {th }}$ century. Since then, numerous techniques have been developed to visualize the spatial organization of the consortium of 
bacteria, fungi, and viruses that live associated with a host-the microbiota ${ }^{1}$. Elucidating the localization of these microbes is essential to determining their function within their animal host. The biogeography of bacteria is important in commensal and pathogenic taxa alike, as proximity to specific locations (e.g., the epithelium), nutritional substrates, and specific microbes may dictate bacterial production of metabolites underlying interspecies and inter-kingdom interactions.

A key structure separating bacteria and the host tissues in several disparate body sites, such as the oral cavity, intestine, or lung, is the mucus - a host-produced layer that both prevents microbial translocation onto the host epithelial cells and serves as a nutritional resource for the microbiota $^{2,3,4}$. Characterizing breaches and changes in this barrier is of key importance, leading to mechanistic insight into host-microbiota interactions that would not be obtained by sequencing alone $e^{5,6,7}$. For example, imaging enabled the discovery that antibiotic exposure can disrupt the mucus layer and microbiota organization ${ }^{7,8}$, and that laxatives may deplete the mucus, correlating with large changes in immune parameters ${ }^{5}$.

This protocol outlines a general framework for fixing, staining, and imaging the microbiota and the host tissue (Figure 1), built upon the work of Johansson and Hansson ${ }^{9}$. While this protocol is modeled in the context of intestinal sections, it can be easily adapted to other tissue types. This protocol enables the processing of either experimental animal or human clinical samples; notes for processing both types of samples have been included. In the example presented here, the host epithelium and luminal bacteria have been simultaneously labeled with 4',6-diamidino-2-phenylindole (DAPI) staining, mucus with the fluorescein (FITC)-conjugated lectin, Ulex europaeus agglutinin I (UEA-1), and a specific bacterial taxon using FISH. FISH probes are usually designed against a taxon's 16S rRNA genes to ensure the high signal from binding a high-copy transcript.

In this example, the probe targets the 16S rRNA of the Muribaculaceae bacterial family (Figure 2). However, the stains are readily substituted with different FISH probes and/or lectins to accommodate the appropriate biological question. Previously validated FISH probes can be found on ProbeBase ${ }^{10}$, an online resource for rRNA-targeted oligonucleotide probes, or on SILVA ${ }^{11}$, a ribosomal RNA database. For the design of new probes, the reader may refer to new pipelines such as HiPR-FISH ${ }^{8}$ or Oligominer ${ }^{12}$. Using this protocol, it is possible to observe the close packing of bacteria in the intestinal lumen and the different features of intestinal mucus throughout the digestive tract. The workflow described here enables the quantitative analysis of the microbiota in the spatial context of its host environment.

\section{Protocol}

All animal experiments and tissue collection described in this protocol were performed in compliance with the Canadian Council on Animal Care (CCAC) guidelines and were approved by the Animal Care Committee at the University of British Columbia. Germ-free Swiss Webster mice were used. All animals were 8-10 weeks of age, both sexes were used, and all mice were co-housed with at least two mice per cage. Animals were euthanized using carbon dioxide with secondary cervical dislocation or cardiac puncture.

\section{Designing an imaging experiment: considerations and sample collection}

1. FISH probe design 
1. If an appropriate FISH probe exists, select a preexisting published one that shows a strong signal in labeled cells compared to the background.

2. Validate new probes in vitro to determine their efficiency of binding to fixed samples of the target bacteria and off-target binding to other bacteria.

3. Check all $16 \mathrm{~S}$ FISH probes to be used against $16 \mathrm{~S}$ sequences from the target organism or against $16 \mathrm{~S}$ rRNA sequencing from the samples to be analyzed to ensure that the expected proportion of positive matches are present, and that probes will not bind non-specifically to other microbiota members.

1. Align the FISH probes against either full-length sequences or amplicon sequence variants using a tool, such as Clustal Omega ${ }^{13}$, to evaluate the potential binding of probes and their targets.

2. In addition to in silico testing, test the accuracy and level of FISH staining of unvalidated probes on pure cultures 8,14 .

NOTE: When staining multiple community members, probes can be used combinatorially (e.g., the combination of a familyspecific probe and a genus-specific probe) if the fluorophores used do not overlap in excitation and emission spectra (unless imaging on a system with the ability to perform linear unmixing). Additionally, specificity may be demonstrated by including specificity controls/scrambled probes or by competition with non-fluorescent probes.

\section{Sample types and tissue collection}

1. Using sharp and clean tools, cut intestinal segments from gnotobiotic Swiss Webster mice for imaging. Minimize disturbing the sample as much as possible, and handle the sections by their edges to avoid affecting the imaging area. Fix the sample as soon as possible after dissection to prevent degradation. NOTE: For animal studies, intact, unflushed intestinal sections are preferred; the membrane will help keep the luminal and mucosal organization intact.

2. Clean tools by wiping them with $70 \%$ ethanol between samples and sites. Obtain at least three biological replicates per intestinal section/biopsy location, taking care to sample consistent intestinal locations, as the mucus and intestinal architecture as well as microbiota changes significantly in different locations.

\section{Tissue sample fixation and infiltration}

1. Fixation

1. Prepare fresh methacarn in a compatible container with the following ratios: $60 \%$ absolute methanol, $30 \%$ chloroform, and 10\% glacial acetic acid. Dispense each of these chemicals in a fume hood. Choose a container that can be vented by cracking open the lid. As methacarn is not compatible with polystyrene, prepare it in a polyethylene container, using glass graduated cylinders/serological pipets for the chloroform and glacial acetic acid.

NOTE: During mixing, fumes are produced and may cause pressure to build up inside the container. It is advisable to keep the container relatively tightly closed once removed from the fume hood if samples are not being actively added. Chloroform is toxic; do not inhale, swallow, or absorb through the skin. Methanol is toxic and highly flammable; do not inhale, swallow, or absorb through the skin. Glacial 
acetic acid is flammable and highly corrosive to the skin and the eyes.

2. After dispensing, close the container, swirl to mix and then vent; repeat this until off-gassing has stopped (and pressure no longer builds up appreciably under the lid).

NOTE: Do not dispose of methacarn in drains; collect and dispose of as hazardous chemical waste as per institutional guidelines. Use a pencil for labeling histology cassettes, as many pen inks will come off in the methacarn solution.

3. Place the intestinal sections in histology cassettes by delicately holding an edge of the tissue with tweezers. Close the cassette and completely submerge it in fresh methacarn solution. Ensure that the solution is not older than a few hours upon immersion of the cassettes.

4. For clinical samples that will be collected in a clinical suite without access to a fume hood, use a polyethylene storage container with a flap cut into the lid that will permit the passage of the histology cassettes. Tape this flap shut when not passing samples to prevent the escape of toxic fumes.

NOTE: It is important to use masking tape to avoid the dissolution of glue onto the container-some types of tape (e.g., plastic packing tape) may not hold up well to the methacarn fumes.

5. Fix samples for a minimum of $3 \mathrm{~h}$ and a maximum of two weeks.

NOTE: Thicker samples may require fixation times longer than $3 \mathrm{~h}$. Fixation time may be chosen to enable simultaneous paraffin infiltration processing for cassettes fixed in different methacarn solutions (e.g., to simultaneously perform paraffin infiltration on groups of samples collected and fixed over two weeks).

2. Paraffin infiltration and embedding

1. Place the paraffin in a heat-resistant container and melt it in an oven at $60{ }^{\circ} \mathrm{C}$ overnight. As paraffin pellets take up more space before melting, ensure that sufficient paraffin is melted to cover all the cassettes.

NOTE: The temperature should not be higher than $60{ }^{\circ} \mathrm{C}$ as it can give rise to artifacts.

2. Wash the tissue by pouring out the liquid in the appropriate waste receptacle and replacing it immediately with the following chemicals.

1. Incubate in absolute methanol for $30 \mathrm{~min}$. Repeat once.

2. Incubate in absolute ethanol for $20 \mathrm{~min}$. Repeat once.

3. Incubate in xylenes for $15 \mathrm{~min}$. Repeat once.

4. Exchange the washes quickly, taking care to avoid letting the cassettes dry. Ensure that each wash fully covers the cassettes in the beaker or container.

NOTE: Xylenes are flammable, and if inhaled, the vapors may depress the central nervous system with potential long-term neurological consequences upon exposure to high concentrations (>200 ppm). Handle xylenes only within a fume hood. As xylenes are hazardous, take care to use the minimum amount necessary to cover the histological cassettes. 
3. During embedding, orient the intestinal segments parallel to the length of the cassette (as opposed to upright) using tweezers to provide longitudinal, transverse sections instead of cross-sectional doughnuts to provide more potential sample area for quantitative imaging (Figure 1B).

4. Open the cassettes slightly ( $1-2 \mathrm{~cm}$ or 0.5 inches) to allow the paraffin to enter without losing the tissue segments. Use double gloves for this step or tweezers to prevent overheating or mild burning of fingers. Submerge and close the cassettes in the container of melted paraffin, placing the container back into the $60^{\circ} \mathrm{C}$ oven. Ensure that the cassettes are filled with paraffin, and no large air bubbles remain

5. After incubation for $2 \mathrm{~h}$ at $60{ }^{\circ} \mathrm{C}$, remove the container from the oven. Using forceps, carefully remove the cassettes and lay them individually on a piece of aluminum foil to cool. Store them at room temperature until ready for embedding and sectioning.

6. Section the paraffin blocks with a microtome, sectioning deep enough into the block that luminal contents are exposed, enabling a longitudinal section of villi and/or crypts in addition to luminal contents and mucus. Take care to replace the blades as fecal material dulls the blade rapidly compared to tissue. Cut sections to $4 \mu \mathrm{m}$ of thickness for optimal sharpness of the images. Transfer the sections to regular uncoated slides.

7. For FISH staining, stain the intestinal sections as soon as possible (i.e., within a few weeks of sectioning) to achieve a strong FISH signal. If omitting FISH staining, lectin + DAPI staining can be performed on less fresh (i.e., months-old) samples without significant loss of signal.

\section{Staining bacteria and host features}

1. Preparation

1. Heat the oven to $60^{\circ} \mathrm{C}$. Pre-warm an empty Coplin jar and enough volume to cover the glass slides in the jar twice with xylenes in a glass bottle, taking care to parafilm around the lid to prevent evaporation of the xylenes. Allow the temperature of the xylenes to reach $60{ }^{\circ} \mathrm{C}$.

NOTE: A standard Coplin jar fits 8 slides, and the slides can be covered with roughly $50 \mathrm{~mL}$ of liquid (may vary between 40 and $60 \mathrm{~mL}$ depending on the brand). Xylene substitutes are less toxic and have been successfully used by other groups for the dewaxing step (step 3.2$)^{8,9}$.

2. If a separate oven is available, pre-warm a hybridization oven to $50^{\circ} \mathrm{C}$. Otherwise, this step may be performed with the same oven used for baking the slides after step 3.2.3.

3. Prepare the FISH hybridization solution: $20 \mathrm{mM}$ Tris$\mathrm{HCl}, \mathrm{pH} 7.4 ; 0.9 \mathrm{M} \mathrm{NaCl} ; 0.01 \%^{26}-0.1 \%^{9}$ (w/ v) sodium dodecylsulfate (SDS) in nuclease-free water. If required, add $5-50 \%(\mathrm{v} / \mathrm{v})$ formamide. Prewarm this hybridization solution at $50{ }^{\circ} \mathrm{C}$ during deparaffinization.

NOTE: Formamide is an amide that acts as a teratogen; handle formamide with gloves and goggles. In small doses and upon exposure to eyes, skin, or mucous membranes, it is irritating. In large amounts, formamide vapor can require medical 
intervention. Formamide can be stored at $100 \%$ in a $-20{ }^{\circ} \mathrm{C}$ freezer.

2. Preparing the slides for staining: deparaffinization

1. Place the slides in the Coplin jar, ensuring that the sections do not come in contact with other slides or the jar, and bake the slides at $60{ }^{\circ} \mathrm{C}$ for $10 \mathrm{~min}$.

2. In the fume hood, fill the Coplin jar with prewarmed xylenes from the oven, taking care not to pour directly on top of the samples and potentially dislodge the tissues. Place the Coplin jar back in the $60{ }^{\circ} \mathrm{C}$ oven. Leave the bottle with the remaining xylenes in the fume hood.

3. Pour the used xylenes into a proper waste container for disposal, taking care not to disturb the tissue sections on the glass slides and using a pair of forceps to keep the slides from falling out of the Coplin jar. Replenish the Coplin jar with the remaining xylenes and incubate for $10 \mathrm{~min}$ at room temperature in the fume hood.

4. Incubate the sections in $99.5 \%$ ethanol for $5 \mathrm{~min}$ at room temperature. After this incubation step, remove the slides from the Coplin jar, wipe the back of the slides on a laboratory wipe or paper towel, and briefly air-dry until the ethanol droplets are gone.

NOTE: Wiping the back of the slides enables better visualization of the drying progress.

\section{Bacterial staining with FISH}

1. Use a liquid blocker or PAP pen to limit the area of liquid expansion by circling the area of each tissue section. Ensure that the ink does not come in contact with the section itself. Create a circle as close to each tissue section as possible without contacting the tissue to minimize the surface area that needs to be covered by the hybridization solution.

2. Prepare the hybridization solution: for every 50 $\mu \mathrm{L}$ of pre-warmed hybridization solution, add 0.5 $\mu g$ probe (e.g., $0.5 \mu \mathrm{L}$ of $1 \mu \mathrm{g} / \mu \mathrm{L}$ probe). Pipette the hybridization solution onto the sections on the slide $(\sim 20 \mu \mathrm{L} /$ section, depending on the section/ circle size). Protect the hybridization solution and the slides from light from this point onward during incubation steps and storage.

3. Ensure that the volume of liquid used covers the entire section upon overlaying with a flexible plastic coverslip. Incubate the slide in a humid chamber to reduce evaporation. Create a humid chamber with a pipette tip box with wipes or paper towels at the bottom that have been soaked with excess hybridization solution or phosphate-buffered saline (PBS) to provide humidity. Incubate the sections at $45-50^{\circ} \mathrm{C}$, depending on the probe set, for $>3 \mathrm{~h}$.

4. Warm up the FISH washing buffer: $20 \mathrm{mM}$ Tris- $\mathrm{HCl}$, $\mathrm{pH} 7.4 ; 0.9 \mathrm{M} \mathrm{NaCl}$ to $50{ }^{\circ} \mathrm{C}$ during the incubation period. Prepare enough washing buffer to cover the slides in the Coplin jar.

5. Remove the plastic coverslips; incubate the slides in FISH washing buffer pre-warmed to $50{ }^{\circ} \mathrm{C}$, placing the Coplin jar back into the $50{ }^{\circ} \mathrm{C}$ oven for $10-20$ min. For thick samples, remove the coverslips in the Coplin jar by adding the buffer and gently dislodging the coverslips to avoid smearing the slice.

4. Staining the mucus and host features

1. Prepare a general DNA/mucus counterstain in PBS: final $10 \mu \mathrm{g} / \mathrm{mL}$ DAPI $+40 \mu \mathrm{g} / \mathrm{mL}$ mucus stain UEA-1. Prepare enough counterstain to cover the spots in 
the absence of a coverslip to avoid damaging tissue with additional coverslips.

NOTE: Covering the spots in the absence of a coverslip will require larger volumes than the $20 \mu \mathrm{L}$ used in 3.3.2. For average-sized sections $(\sim 10 \mathrm{~mm}$ diameter), $200 \mu \mathrm{L}$ is enough to cover one spot; test this volume beforehand on a dummy slide.

2. Remove the FISH washing buffer and replace it with PBS in the Coplin jar. Immediately after refilling the Coplin jar with PBS, decant the PBS.

3. Remove the slides from the jar and pipette the counterstain on top of the section while ensuring to not touch the tissue with the pipette tip. Cover the entire section with a bubble. Incubate at $4{ }^{\circ} \mathrm{C}$ for 45 $\min$ in the dark.

4. Wash the stains 3 times quickly with fresh PBS: while holding the slides in place using tweezers, pour out the PBS in a sink and immediately refill with fresh PBS.

5. Wiping the back of the slides against a wipe or paper towel, let most of the PBS evaporate off the sections, aided by a vacuum line connected to a pipette tip. Once again, avoid touching the section with the pipette tip.

6. Mount the sections using a mounting medium (without DAPI) and let it set at room temperature covered by glass coverslips, ensuring that the coverslips are flat and there are no air bubbles in the mounting medium.

7. Affix the coverslips to the slide by painting along the edges of the coverslip with clear nail polish, taking care to stay away from the edge of the slide to ensure that the slide will sit level in the microscope slide holder during imaging.

8. Store the slides at $4{ }^{\circ} \mathrm{C}$ in the dark for a few weeks before the fluorescence is depleted.

\section{Imaging and image analysis}

1. Visualizing the stains

1. Select an area of the tissue that contains both tissue and lumen to image the microbiota-host interface. For quantitative imaging of mucus thickness and luminal biogeography, select fields of view where the slices of the epithelial villi and/or crypts are longitudinal and not cross-sectional. Avoid areas with large gaps between the mucus and the epithelium to avoid sectioning artifacts.

2. Locate tissue and correct the focal plane, utilizing the DAPI signal for locating and focusing to avoid photobleaching of the FISH fluorescence signal.

3. Adjust the imaging settings. To visualize the bacterial DAPI stain, increase the laser power and gain to the point where the DAPI signal from epithelial cells is oversaturated or "blown out."

NOTE: Do not oversaturate excessively, as this will lead to photobleaching and visual artifacts in the nuclei that cannot be recovered.

4. For all other channels, use the minimum amount of laser power that will provide a clear signal above the background, and be careful not to oversaturate.

NOTE: This is especially important when performing tile-scans, as photobleaching will be problematic when the overlap between tiles is imaged.

5. Use $40 x$ or higher magnification to image individual bacteria. 
6. Acquire the images.

1. Acquire tile scans to obtain quantitative data on mucus thickness and spatial distribution of microbes within the lumen. Ensure 15\% overlap between the tiles and check for vignetting/ uneven illumination, which may be exacerbated by $<1 x$ zoom. When setting up a tile scan, pay close attention to whether the tissue is in focus in all the tiles, as blurry/out-of-focus images cannot be analyzed (Figure 3B).

2. If possible, minimize the number of tiles needed to image a given length of epithelium by rotating the scan area so that the epithelium and mucus layer are parallel to the tile and not at an angle. Otherwise, find a section of the epithelium that is parallel or perpendicular to the imaging area to achieve a similar effect.

\section{Representative Results}

To investigate the localization of specific gut commensal bacteria in the mouse intestine, germ-free Swiss Webster mice that had been colonized with individual bacterial isolates were used in this study. For this experiment, the bacterial species labeled were i) a human isolate of the Muribaculaceae family ${ }^{5}$, Muribaculum intestinale, an abundant and prevalent family of bacteria in the murine microbiota $^{15}$, as well as ii) Bacteroides thetaiotaomicron, a genetically tractable and common gut bacterium. Following two weeks of equilibration, the mice were euthanized, and a segment of the colon containing a fecal pellet was sectioned and fixed in freshly prepared methacarn. After two days of fixation, the sections were dehydrated by processing through methanol, ethanol, and xylenes, infiltrated with paraffin, embedded, and then sectioned to luminal 4 $\mu \mathrm{m}$ slices. These samples were then stained with a FISH probe (3' Cy3-tagged) specific for the Muribaculum isolate, designed using Oligominer software ${ }^{12}$, and checked for secondary and tertiary structure and minimal non-specific binding using NuPACK and mathFISH ${ }^{16,17}$. The samples were also counterstained with the Rhodamine-bound lectin UEA-1 (which stains fucosylated glycans in mucus) and DAPI. The stained sections were imaged using a $40 x$ oil objective and super-resolution module. 


\section{A}

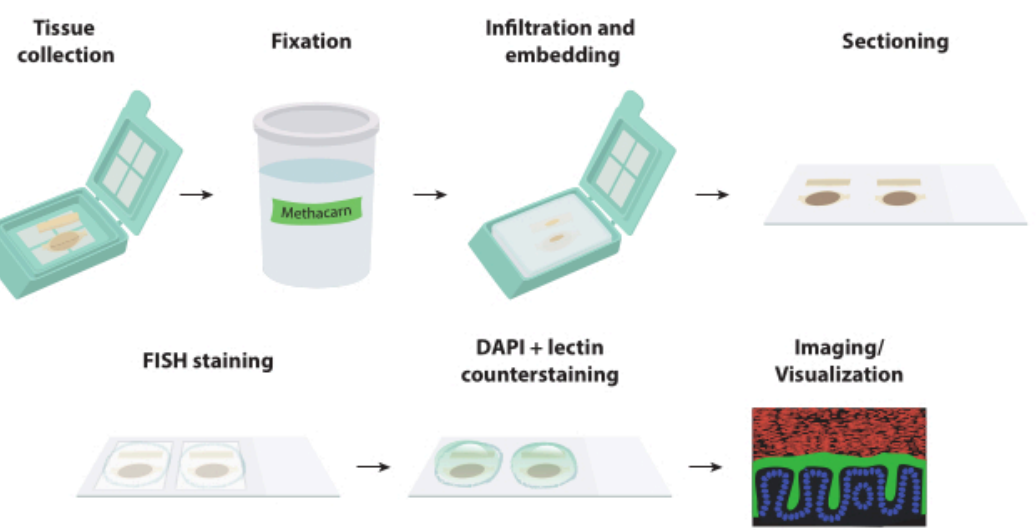

B

C
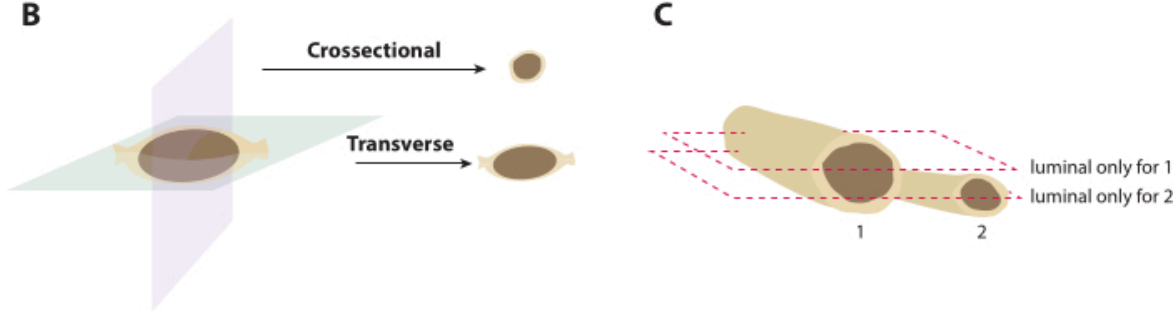

Figure 1: Workflow of visualization of the microbiota-host interface in the intestine. (A) An illustrated workflow for the pipeline. (B) The two sectioning planes will yield either transverse sections (preferred for this pipeline) or cross-sectional "doughnuts." (C) Embedding tissues of very different thicknesses may result in slices that are only optimal for one tissue or another. Abbreviations: FISH = fluorescence in situ hybridization; DAPI = 4',6-diamidino-2-phenylindole. Please click here to view a larger version of this figure. 
A

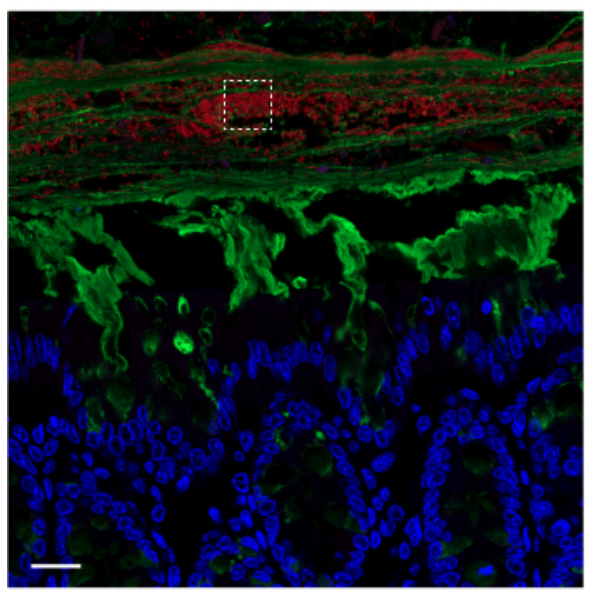

C
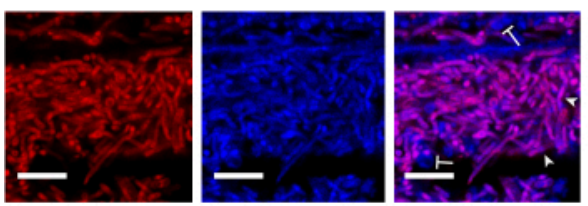

B

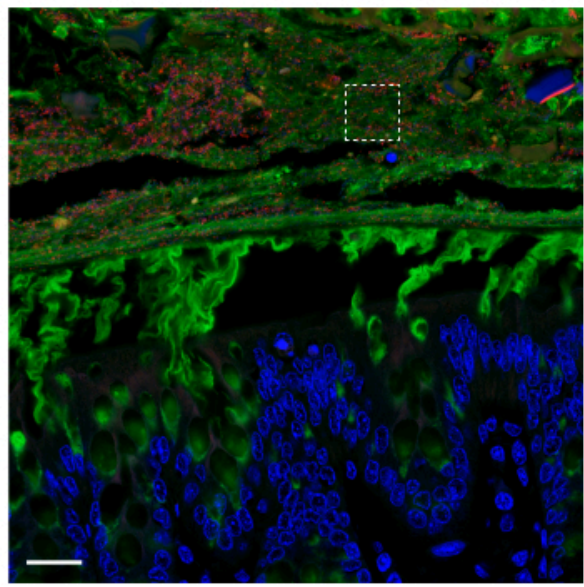

D
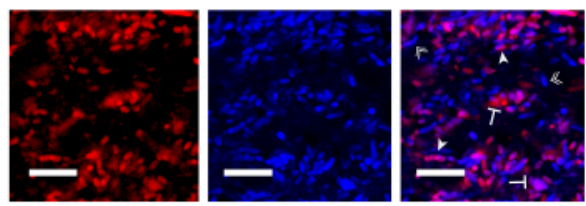

Figure 2: Imaging the colon shows the localization of Muribaculum intestinale relative to mucus in a gnotobiotic mouse model. Sections were stained with DAPI (blue), Muribaculaceae FISH probe (red), and UEA-1 (green). (A) Distal colon of mouse mono-colonized with Muribaculum intestinale and (B) distal colon of mouse bi-colonized with Muribaculum intestinale and Bacteroides thetaiotaomicron. Scale bar $=20 \mu \mathrm{m}$. (C and D) Cy3-FISH (left), DAPI (middle), and combined Cy3-FISH + DAPI channels for luminal inset portions of $(\mathbf{A})$ and $(\mathbf{B})$, respectively. Scale bar $=5 \mu \mathrm{m}$. In $(\mathbf{A})$ and $(\mathbf{C})$, all bacteria-shaped and bacteria-sized DAPI signals are labeled with Cy3 (single arrowheads), and in (B) and (D), in addition to these Cy3- and DAPI-double-positive cells (single arrowheads), there are DAPI-stained bacterial cells that are Cy3-negative (double arrowheads), as expected for mono- and bi-colonization states. With the exception of longer filamentous bacteria, larger (>4 $\mu \mathrm{m}$ in length) DAPI-positive structures (blunt arrows) are plant material or nuclei from host cells. Abbreviations: $\mathrm{FISH}=$ fluorescence in situ hybridization; DAPI = 4',6-diamidino-2-phenylindole. Please click here to view a larger version of this figure. 
A

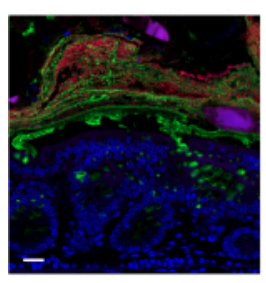

C

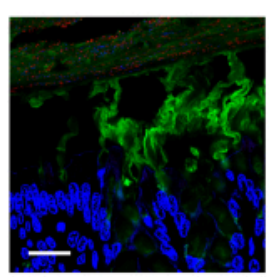

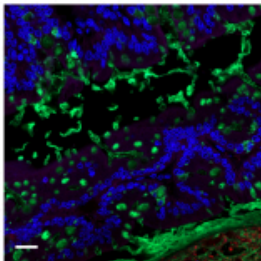

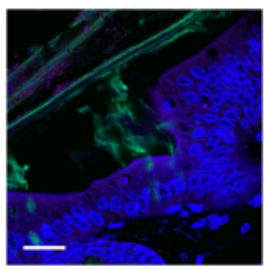

B

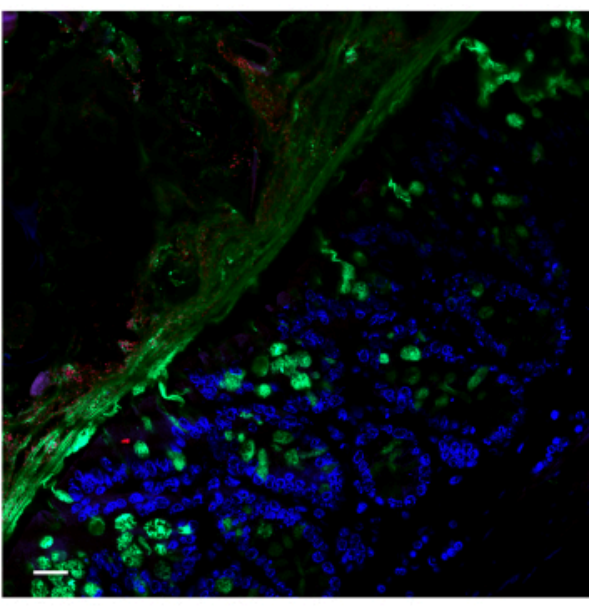

Figure 3: Common problems. (A) Shallow sectioning will not provide luminal slices of the intestine. (Left) An intestinal segment that has been sectioned at a depth that provides a view of the lumen as well as longitudinal views of the epithelium. (Right) An intestinal segment that has been sectioned too shallowly, revealing only cross-sections of crypts and no constant mucus layer or bacteria. (B) Uneven tissue/coverslip coverage may result in blurry and unevenly illuminated tile-scans. A tilescan was set up with positions that were out of focus (top right corner). (C) Example of normal background (left) and high background (right). In this example, for the DAPI signal-note the signal coming from the epithelium, outside the nuclei. All scale bars = $20 \mu \mathrm{m}$. Abbreviation: DAPI = 4',6-diamidino-2-phenylindole. Please click here to view a larger version of this figure.

\section{Discussion}

The protocol described above provides a reproducible method to visualize the host-microbiota interface. These assays have considerably benefited from protocol development, starting from the optimization of FISH labeling $^{18}$ to mucus preservation and imaging 9 . Fixation and embedding also provide useful storage of samples; furthermore, paraffin-infiltrated cassettes can be mailed without any restrictions, as the samples are completely fixed and inert.

\section{Significance and alternative methods}

The combination of FISH and mucus staining enables the analysis of microbiota composition at specific tissue locations and the interaction between individual bacteria and the host. Alternative techniques that involve the analysis of specific sites within the microbiota are usually unable to explore the single-cell nature of these interactions, such as in the case of laser capture microdissection coupled with sequencing ${ }^{19}$. However, sequencing-based techniques have the advantage of being able to capture the genetic make-up of the microbiota at a finer level and more broadly than $16 \mathrm{~S}$ rRNA probes.

A pitfall of the protocol presented is that it provides a single-time snapshot of the microbiota in relation to the 
host. For real-time imaging in live animals, special imaging techniques are required to facilitate the acquisition of a fluorescence signal in deep tissue (e.g., intravital two-photon microscopy and light-sheet fluorescence microscopy ${ }^{20,21}$ ). In these methods, the model organism is either colonized with bacteria that are stained with molecules that bind to their envelope ${ }^{20}$ or genetically modified bacteria that harbor fluorescent proteins ${ }^{21}$. In the latter case, the maturation of fluorescence proteins is a key issue as most standard fluorescent proteins require oxygen to emit light. Therefore, model organisms that have at least nanaerobic environments (nanomolar concentration of oxygen) are required, such as the aerobic zebrafish gut ${ }^{21}$.

In this protocol, methanol-Carnoy is used as a fixative instead of paraformaldehyde or formalin because it does not contain water. This prevents hydration, dehydration, and collapse of the mucus layer during processing and facilitates accurate measurements of mucus thickness. While methacarn fixation and paraffin embedding have become a standard in the field, different fixatives and embedding techniques have been investigated to optimize mucus preservation ${ }^{9,22}$, with some studies indicating that resins may be superior to paraffin embedding 22 . Another important limitation to paraffin embedding and methacarn fixation is the loss of fluorescent protein fluorescence, which can be avoided by using alternative fixatives (e.g., formalin or paraformaldehyde (PFA)) or modified embedding techniques ${ }^{23}$. Each fixative has benefits and drawbacks, and the choice of the fixative depends on the imaging priorities. Imaging modalities can be combined if biological replicates are fixed in either PFA and methacarn and images are obtained from both samples.

FISH has been combined with immunofluorescence in isolated instances with specific anti-Muc2C3 rabbit polyclonal antibodies $^{9,24}$. These results have not been widely reproduced with other Muc2 antibodies, indicating that the choice of antibodies may be critical in the success of the FISH-immunofluorescence combination. The conditions for successful antibody staining for a given antibody may not permit retention of FISH staining.

\section{Troubleshooting}

In this protocol, sample collection, sectioning, and staining represent critical steps to prevent the creation of imaging artifacts. For example, pressing on the tissue upon collection and before embedding can lead to mislocalization of the microbiota and affect mucus quality. Another important consideration is to avoid combining segments of very different thicknesses in a single cassette, such as a relatively empty piece of the small intestine and a pellet within the distal colon. The different thicknesses lead to difficulties in imaging: after embedding, transverse sectioning at a specific depth for one segment may be at the wrong depth for imaging for the other one (e.g., the lumen will be at different depths for each tissue) (Figure 1B,C and Figure 3A). Furthermore, for imaging animal model stool pellets, they may be wrapped in peritoneal membrane ${ }^{24}$. In this technique, a small section of freshly isolated peritoneum is gently folded around the stool and preserves the structure of the pellet during the wash steps outlined in the protocol.

Unlike processing animal tissue with contents, imaging human intestinal samples presents some challenges. Specifically, luminal contents and mucosal lining will likely be disrupted by the colonoscopy bowel preparation usually performed before intestinal biopsies or resection procedures. Additionally, when biopsies are collected, it is helpful to note the tissue orientation to aid in the identification of specific features (e.g., lumen vs. submucosa) in the absence 
of intestinal contents. Pre-embedding with 3\% agar and/or agar:gelatin mixtures may be useful in maintaining tissue polarity $^{25}$; however, there are issues with the dehydration and sectioning of agar, as reported in the literature, and processing times may need to be altered.

A key aspect to achieving good FISH staining comes from adjusting the formamide concentration for specific FISH probes. Formamide will control the hybridization stringency of FISH probes to their targets. It is important to ensure that a) the proper formamide concentration is chosen for staining a given community, and b) that a suitable formamide concentration is even possible for the probe combination that is being utilized-this may affect the optimal probe choice when utilizing multiple probes. Websites such as mathFISH (http:// mathfish.cee.wisc.edu/) may help with calculating the proper formamide concentrations for a given probe. In the absence of information about appropriate formamide concentrations, this protocol can be tested with $0 \%$ and $10 \%$ formamide.

Sectioning the embedded samples requires cutting the block to a sufficient depth to obtain luminal slices, as sectioning too shallowly into a block will result in a cross-sectional view of the villi/crypts with no luminal contents (Figure 3A). Stain the samples soon after sectioning for optimal FISH signal, and use fresh DAPI (or DAPI stored at $-20{ }^{\circ} \mathrm{C}$ ) to resolve background issues (Figure 3C). FISH staining of sections that are more than a month-old may result in poorer/inconsistent staining.

If the tissue or coverslip is not perfectly flat with respect to the slide, the sample may not be in focus at every position within a tile scan (Figure 3B), leading to wasted effort and potential photobleaching. This can be solved by taking smaller tile scans in which all positions have been verified to be in focus. Sectioning with dull blades can also lead to the detachment of the mucus and luminal contents, which appear as large dark areas while imaging. Finally, the evaporation of the solution or bubbles during the hybridization step can lead to uneven staining of the FISH probes in the tissue.

Another critical parameter that affects the efficiency of the FISH probe binding is the competition between different probes. A useful check to verify that the FISH probe is labeling bacteria is to examine the colocalization of the signal from the FISH probe with DAPI (Figure 2C,D). In samples that require the use of multiple rRNA probes, adjusting parameters such as hybridization temperature, probe concentration, and formamide becomes critical to ensure that the probes are binding to the correct species efficiently ${ }^{18}$.

\section{Notes about imaging}

FISH-stained bacteria are best visualized using a confocal microscope and an objective of at least 40x magnification. While $63 x$ magnification is preferred to image bacteria at the single-cell level, 40x magnification can also be used by maximizing the digital zoom or employing a super-resolution system. Systems equipped with super-resolution capabilities may also provide sub-cellular resolution of individual bacterial cells. Lower magnification objectives may be utilized to get a general sense of bacterial localization and mucus thickness.

Acquiring 16-bit images instead of 8-bit images is also highly recommended, as the higher dynamic range will help to capture the wide range of the DAPI signal from the extremely bright nuclei of the epithelium and the relatively weak signal of luminal bacteria. Aside from using the imaging setup described above, an important imaging consideration is the choice of fluorophores. For best separation between bacterial types, ensure that the fluorophores used do not overlap in excitation and emission spectra (unless imaging on a system with the ability to perform linear unmixing). Additionally, 
probes can be used in combination (e.g., the combination of a family-specific probe and a genus-specific probe) to identify additional community members. If using linear unmixing or unvalidated probes, it is essential to test the accuracy and level of FISH staining on pure cultures ${ }^{8,14}$.

Once the images are collected, multiple tools are available for the quantitative analysis of the host-microbiota interface, such as BacSpace ${ }^{26}, \mathrm{HiPR}_{\mathrm{F}} \mathrm{FSH}^{8}$, and other segmentation tools ${ }^{27}$. BacSpace is a MATLAB software that allows the segmentation of the tissue and luminal components and the removal of plant material from images ${ }^{26}$. The program also provides analysis of mucus thickness in $2 \mathrm{D}$ and quantification of spatial distribution based on pixel distances in different channels $^{26}$. Conversely, the HiPR-FISH Image Analysis software allows the segmentation of FISH-stained cells ${ }^{8}$. Finally, other bacterial segmentation tools that have been optimized for in vitro experiments ${ }^{27}$ could also be adapted to this application.

\section{Conclusion}

In situ imaging enables the quantitative analysis of individual microbial taxa in the context of other community members and the various microenvironments created by diet, mucus, and host epithelial crypts. The interaction between organisms dictates the biology of host-associated microbial systems and provides an essential analysis of change in these structures during perturbation that has implications for health. Notably, the ability to image the microbiota in situ through the protocol described above provides an incredible window into the biogeography of the microbiota in relation to the animal host.

\section{Disclosures}

The authors have no conflicts of interest.

\section{Acknowledgments}

The authors would like to thank Dr. Kristen Earle for invaluable technique development, Amber Hann for troubleshooting help, Dr. Jen Nguyen and Deanna Pepin for critical review of the manuscript, and Dr. Hesham Soliman and the Rossi lab at the University of British Columbia for providing microscope access. The authors acknowledge support from CIHR Team Grant: Canadian Microbiome Initiative 2 (C.T.), Crohn's and Colitis Canada (to C.T. and K.M.N.), CIFAR (to C.T. and K.M.N.), Michael Smith Foundation for Health Research Scholar Award (to C.T.), the Weston Foundation: Weston Family Microbiome Initiative (to C.T.), Paul Allen Distinguished Investigator Award (to C.T.).

\section{References}

1. Tropini, C., Earle, K. A., Huang, K. C., Sonnenburg, J. L. The gut microbiome: connecting spatial organization to function. Cell Host and Microbe. 21 (4), 433-442 (2017).

2. Hansson, G. C., Johansson, M. E. V. The inner of the two Muc2 mucin-dependent mucus layers in colon is devoid of bacteria. Gut Microbes. 1 (1), 51-54 (2010).

3. Johansson, M. E. V. et al. Normalization of host intestinal mucus layers requires long-term microbial colonization. Cell Host and Microbe. 18 (5), 582-592 (2015).

4. Jakobsson, H. E. et al. The composition of the gut microbiota shapes the colon mucus barrier. EMBO Reports. 16 (2), 164-177 (2015).

5. Tropini, C. et al. Transient osmotic perturbation causes long-term alteration to the aut microbiota. Cell. 173 (7), 1742-1754.e17 (2018).

6. Desai, M. S. et al. A dietary fiber-deprived gut microbiota degrades the colonic mucus barrier and enhances 
pathogen susceptibility. Cell. 167 (5), 1339-1353.e21 (2016).

7. Ng, K. M. et al. Recovery of the gut microbiota after antibiotics depends on host diet, community context, and environmental reservoirs. Cell Host and Microbe. 26 (5), 650-665.e4 (2019).

8. Shi, H. et al. Highly multiplexed spatial mapping of microbial communities. Nature. 588 (7839), 676-681 (2020).

9. Johansson, M. E. V., Hansson, G. C. Preservation of mucus in histological sections, immunostaining of mucins in fixed tissue, and localization of bacteria with FISH. Methods in Molecular Biology. 842, 229-235 (2012).

10. Greuter, D., Loy, A., Horn, M., Rattei, T. ProbeBasean online resource for rRNA-targeted oligonucleotide probes and primers: New features 2016. Nucleic Acids Research. 44 (D1), D586-D589 (2016).

11. Quast, C. et al. The SILVA ribosomal RNA gene database project: Improved data processing and webbased tools. Nucleic Acids Research. 41 (D1), D590D596 (2013).

12. Beliveau, B. J. et al. OligoMiner provides a rapid, flexible environment for the design of genome-scale oligonucleotide in situ hybridization probes. Proceedings of the National Academy of Sciences of the United States of America. 115 (10), E2183-E2192 (2018).

13. Madeira, F. et al. The EMBL-EBI search and sequence analysis tools APIs in 2019. Nucleic Acids Research. 47 (W1), W636-W641 (2019).

14. Mark Welch, J. L., Hasegawa, Y., McNulty, N. P., Gordon, J. I., Borisy, G. G. Spatial organization of a model 15-member human gut microbiota established in gnotobiotic mice. Proceedings of the National Academy of Sciences of the United States of America. 114 (43), E9105-E9114 (2017).

15. Lagkouvardos, I. et al. Sequence and cultivation study of Muribaculaceae reveals novel species, host preference, and functional potential of this yet undescribed family. Microbiome. 7 (1), 28 (2019).

16. Zadeh, J. N. et al. NUPACK: Analysis and design of nucleic acid systems. Journal of Computational Chemistry. 32 (1), 170-173 (2011).

17. Yilmaz, L. S., Parnerkar, S., Noguera, D. R. MathFISH, a web tool that uses thermodynamics-based mathematical models for in silico evaluation of oligonucleotide probes for fluorescence in situ hybridization. Applied and Environmental Microbiology. 77 (3), 1118-1122 (2011).

18. Huber, D., Voith von Voithenberg, L., Kaigala, G. V. Fluorescence in situ hybridization (FISH): History, limitations and what to expect from micro-scale FISH? Micro and Nano Engineering. 1, 15-24 (2018).

19. Wang, Y. et al. Laser capture microdissection and metagenomic analysis of intact mucosa-associated microbial communities of human colon. Applied Microbiology and Biotechnology. 88 (6), 1333-1342 (2010).

20. Geva-Zatorsky, N. et al. In vivo imaging and tracking of host\&ndash;microbiota interactions via metabolic labeling of gut anaerobic bacteria. Nature medicine. 21 (9), 1091-1100 (2015).

21. Jemielita, M. et al. Spatial and temporal features of the growth of a bacterial species colonizing the zebrafish gut. mBio. 5 (6), e01751-14 (2014). 
22. Hasegawa, Y., Mark Welch, J. L., Rossetti, B. J., Borisy, G. G. Preservation of three-dimensional spatial structure in the gut microbiome. PLoS ONE. 12 (11), e0188257 (2017).

23. Zhanmu, O. et al. Maintenance of fluorescence during paraffin embedding of fluorescent protein-labeled specimens. Frontiers in Neuroscience. 13, 752 (2019).

24. Bergstrom, $\mathrm{K}$. et al. Proximal colon-derived Oglycosylated mucus encapsulates and modulates the microbiota. Science. 370 (6515), 467-472 (2020).

25. Jones, M. V., Calabresi, P. A. Agar-gelatin for embedding tissues prior to paraffin processing. BioTechniques. 42 (5), 569-570 (2007).

26. Earle, K. A. et al. Quantitative imaging of gut microbiota spatial organization. Cell Host and Microbe. 18 (4), 478-488 (2015).

27. Jeckel, H., Drescher, K. Advances and opportunities in image analysis of bacterial cells and communities. FEMS Microbiology Reviews. fuaa062 (2020). 